\title{
Automatic Generation of Drug Concentration Gradient by a Modified Ink-jet Printer and its Application in Drug Screening
}

\author{
Zongzheng Chen ${ }^{1,3}$, Jiu Deng ${ }^{2}$, Zhengzhi $\mathrm{Wu}^{1}$ and Yong Luo*2 \\ ${ }^{1}$ The First Affiliated Hospital of Shenzhen University, Shenzhen Second People's Hospital, China \\ ${ }^{2}$ Department of Chemical Engineering, Dalian University of Technology, China \\ ${ }^{3}$ Integrated Chinese and Western Medicine Postdoctoral research station, Jinan University, China
}

*Corresponding author: Yong Luo, Department of Chemical Engineering, Dalian University of Technology, China.

To Cite This Article: Yong Luo. Automatic Generation of Drug Concentration Gradient by a Modified Ink-jet Printer and its Application in Drug Screening. Am J Biomed Sci \& Res. 2019 - 5(1). AJBSR.MS.ID.000876. DOI: 10.34297/AJBSR.2019.05.000876

Received: 制 August 30, 2019; Published: 制 September 06, 2019

\begin{abstract}
Manual preparation of drug concentration gradient is labor-intensive and limits the efficiency of a drug screening to some extent, especially at large scale. In this study, we reported an automatic method to generate drug concentration gradient in 24-well plate. We loaded drug solution in a modified hot ink-jet printer, designed printing pattern in the WORD software, customized the concentration gradient in the WORD software by RGB coding, and finally printed the drug concentration gradient in the 24-well plate. A drug concentration gradient can be automatically generated within $5 \mathrm{~s}$ in arbitrary pattern. Based on this flexible technology, we developed an ink-jet printer-based drug screening assay. Concentration gradient of 5-fluorouracil, as well as Mcf-7 cells, were printed in the 24-well plate, and the inhibition rate of Mcf-7 cells was measured by MTT method. This was the first time that hot inkjet printing technology was used in drug screening.
\end{abstract}

Keywords: Bioprinting; Drug screening; Concentration gradient; Ink-jet printer; RGB

\section{Introduction}

Preparation of a concentration gradient of drug candidate is a prerequisite to study cell-drug interaction quantitatively. Currently, this step is manually operated in the laboratory, which is labor intensive and annoying. Automation is desirable in practice. Derringer et al. developed an automatic method to generate concentration gradient [1], and Ye et al. [2] used this method in drug screening [2,3], however, this method was only applicable in microfluidic device, not in 96-well or 24-well plate, thus its applications were limited. Previously, we established drug screening device based on biomedical engineering methods [4]. In this study, we developed a method to generate concentration gradient in plate automatically, using normal commercial ink-jet printer and WORD software. We also used the same printer to print the cell suspension, thus we finally developed a drug screening assay based on hot ink-jet printing. Hot ink-jet printing technique is useful for printing cells in biomedical engineering. It works by the principle in which the heating element turns the "biological inks" into bubbles at bottom of the nozzle instantly and the pressure generated makes a certain amount of inks free from the surface tension there and pushed from the nozzle. As an approach for cell printing, hot ink-jet printer has been used in tissue engineering [5], biological microarray construction [6], stem cell research [7] and gene transcription [8]. However, there are few reports in its application in drug screening at cellular level.

In this paper, a hot ink-jet printer was custom-modified as an automatic dispensing tool for the engineering of cell arrays and generation of concentration gradient of drugs in a 24-well plate. By means of this apparatus, cell/drug composite arrays can be automatically generated for drug screening. RGB value was used as the output gauge of drug concentrations for the first time. A Mcf-7 cell/5-fluorouracil composite array was engineered to demonstrate the applicability of the hot ink-jet printer in the drug screening.

\section{Experimental}

\section{Materials}

Hoechst 33342 fluorescence dye and 3-(4,5-dimethylthiazol-2yl)-2,5-diphenyltetrazolium bromide (MTT) were purchased from Kubolai inc. Beijing. Propidium iodide was obtained from Jingchun technology inc. Shanghai. 5-fluorouracil was purchased from SigmaAldrich, United Kingdom. Phosphorate buffer saline (pH7.4) was prepared and sterilized in lab. DMEM cell culture medium and fetal bovine serum from HyClone, New Zealand, was used in this study. Methanol of HPLC grade from local company was used. Ultrapure water was obtained with Millipore water purification system. 


\section{Instruments}

Canon PIXMA ip 1980 ink-jet printer with PG830 ink box from Canon, Japan, was modified in this study. Inverted fluorescence microscope IX71 from Olympus, Japan, was used. Agilent 1200 HPLC was from Agilent, USA. Synergy H1 microplate reader was from BioTek, USA (Figure 1). A Canon PIXMA ip 1980 printer and a Canon PG830 ink box for biological printing after modification. The white plate was a 24-well plate used as "paper".

\section{Modification of the Printer and the Cartridge}

A Canon PIXMA ip 1980 hot-injet printer was modified as follows the front cover, paper support, rear tray, paper thickness lever and paper guide were dissembled sequentially while cartridge holder and paper sensor being kept intact. The printer after modification was shown as in Figure 1 (left part). Before use, the body of the printer was sterilized and kept inside an ultraviolet-lighted laminar hood overnight. A Canon PG830 ink box was modified as follows: after the top cover of the ink box was ripped from the main body of the box, the sponge inside the ink box was discarded and the metal filter at the bottom of the box was dismantled with the ink reservoir exposed, followed by a thorough cleanup. The ink box after modification was shown as in Figure 1 (right part). Prior to use, the box was emerged in $75 \%$ ethanol and air dried in a laminar airflow, followed by being exposed with ultraviolet light for at least 30 mins.
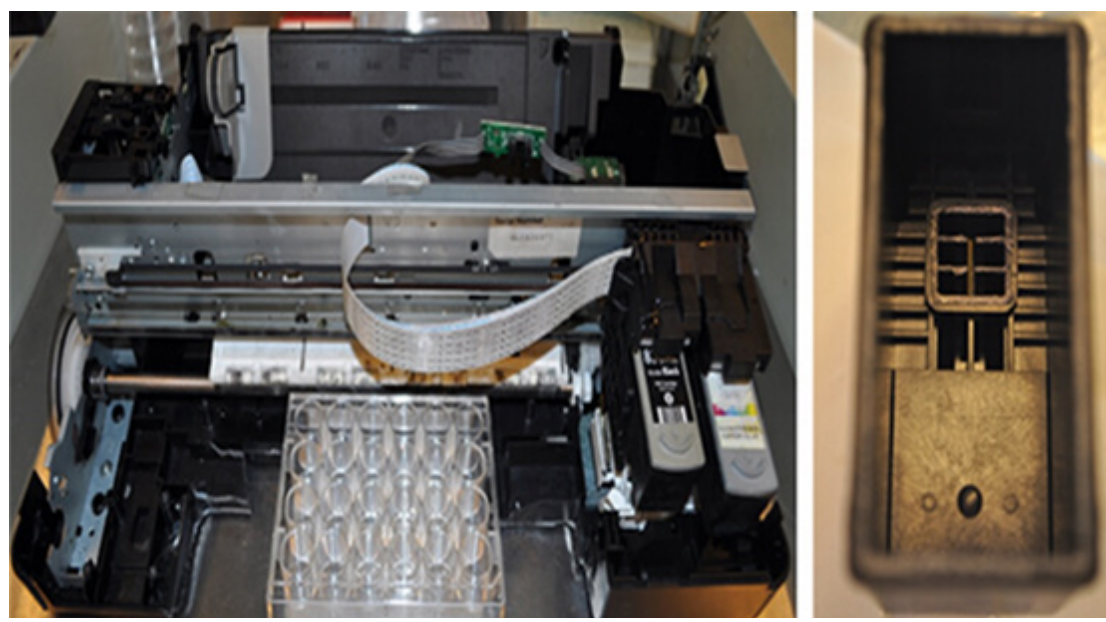

Figure 1: A Canon PIXMA Ip 1980 Printer and a Canon PG830 Ink Box.

\section{Printing Concentration Gradient of 5-Fluorouracil in the} 24-Well Plate

$10 \mathrm{ml} 8 \mathrm{mg} / \mathrm{ml} 5$-fluorouracil solution was precisely obtained when 0.08g 5-fluorouracil was dissolved in PBS solution in a $10 \mathrm{ml}$ volumetric flask and filtered through a $0.22 \mu \mathrm{m}$ diameter membrane to remove possible bacteria. This working solution was loaded in the ink box. The printing pattern was designed in the WORD software with designated RGB values. Clicked the "print" button in the WORD software and the concentration gradient of 5 -fluorouracil was automated created in the 24-well plate within $5 \mathrm{~s}$. We collected the drug solution printed in each well and analyzed their concentrations with HPLC. In a typical setup, a SB-C18 HPLC column (4.6mm diameter, $250 \mathrm{~mm}$ long) was used and the sample was detected by ultraviolet absorption with a single wavelength of $265 \mathrm{~nm}$. The temperature of the column was set at $35^{\circ} \mathrm{C}$. The volume of each sample was $10 \mu \mathrm{l}$ and the mobile phase was made of methanol and water $(5: 95)$ with a flow rate of $1.0 \mathrm{ml} / \mathrm{min}$.

\section{Preparation and Printing of Cell Suspension}

Mcf-7 cells of the third generation with a high viability was chosen as the main raw material for preparation of the cell suspension. Prior to being loaded into the ink box, a Mcf-7 cell suspension were prepared by pancreatic digestion, centrifuging, collection, counting and re-suspending. The Mcf-7 cell suspensions were prepared at four different concentrations, $3 \times 106,6 \times 106$, $9 \times 106$ and $1.2 \times 107 / \mathrm{ml}$. Following the pattern of a round spot with the diameter of $5 \mathrm{~mm}$, the cell suspension with different densities were printed into a 24-well plate with the modified printer as above. Observation was made with an inverted microscope equipped with a X4 zoom objective.

\section{Counting of Printed Cell Numbers and Viability Analysis}

$400 \mu \mathrm{l} 10 \%$ NCBS culture media was added into the plate loaded with the printed Mcf-7 cells to keep their viability. And $10 \mu \mathrm{l}$ $0.4 \mathrm{mg} / \mathrm{ml}$ Hoechst 33342 solution was added into the plate at $37^{\circ} \mathrm{C}$ for $35 \mathrm{mins}$ in the dark, followed by the addition of $10 \mu \mathrm{l} 0.2 \mathrm{mg} /$ $\mathrm{ml}$ PI solution for another 10 mins. Then the cell suspension in the plate were collected and centrifuged. The cells from the bottom of centrifuge tube were collected and washed with PBS solution for 3 times. Then the cells washed were re-suspended in $1 \mathrm{ml}$ cell culture medium to form a new cell suspension. $20 \mu \mathrm{l}$ new cell suspension was pipetted out and added dropwise onto a cell counter. Counting of cell number and observation of cells were conducted with an inverted fluorescence microscope with a X 10 zoom objective.

\section{Procedure of Drug Screening}

Mcf-7 cells with a high viability was selected for construction of cell/drug arrays. The Mcf-7 cell suspension were prepared by pancreatic digestion, centrifuging, collection, counting, and resuspension with a concentration of $9 \times 106 / \mathrm{ml}$. The Mcf-7 cell suspension was loaded into sterilized a PG830 ink box and printed into 5-mm-diameter round spots in a 24-well plate to form a Mcf-7 
cell array. $400 \mu \mathrm{l} 10 \%$ NCBS culture media was immediately loaded into the plate. After being cultured at $37^{\circ} \mathrm{C}$ under $5 \% \mathrm{CO}_{2}$ for $24 \mathrm{hrs}$, $8 \mathrm{mg} / \mathrm{ml} 5$-fluorouracil solution were printed into the plate which were already loaded with Mcf-7 cells, with different RGB codes. As a result, cell/drug composite arrays $(n=3)$ were formed. After being at $37^{\circ} \mathrm{C}, 5 \% \mathrm{CO}_{2}$ for another $48 \mathrm{hrs}, 40 \mu \mathrm{l} 5 \mathrm{mg} / \mathrm{ml}$ MTT solution were loaded into the plate and incubated at $37^{\circ} \mathrm{C}$ for $4 \mathrm{hrs}$. Then the supernatant was discarded and $500 \mu \mathrm{l}$ DMSO was added. The plate was shaken for $15 \mathrm{mins}$ to achieve DMSO thoroughly dissolved. Then $200 \mu \mathrm{l}$ supernatant was collected and transferred into a 96-well plate and the adsorption under $490 \mathrm{~nm}$ wavelength was recorded.

\section{Results}

\section{Printed Concentration Gradient of 5-Fluorouracil}

The printing pattern was designed in WORD software, as shown in Figure 2. Each round spot, corresponding to a well in 24-well plate in location, was $5 \mathrm{~mm}$ in diameter. These round spots varied in RGB values. We printed drugs following the pattern in Figure 2 and measured the concentration of drug in each well. We plotted the drug concentration in each well, as shown in Figure 3. This technology has five advantages compared to manual method

a) It is automatic;

b) We can obtain arbitrary concentration gradient by customizing the RGB values (Figure $3 \mathrm{~A}-\mathrm{D}$ );

c) The RGB code value was linearly related to the concentration of printed 5-fluorouracil. This can be expressed as $\mathrm{Y}$ $=-0.7691 \mathrm{X}+53.975$ with R2 being equal to 0.9878 (Figure $3 \mathrm{~A}$ );

d) It is fast, and a concentration gradient can be generated within $5 \mathrm{~s}$;

e) THE hot inkjet printer was cheaper than a micropipette which is used for manual preparation. (Figure 2)

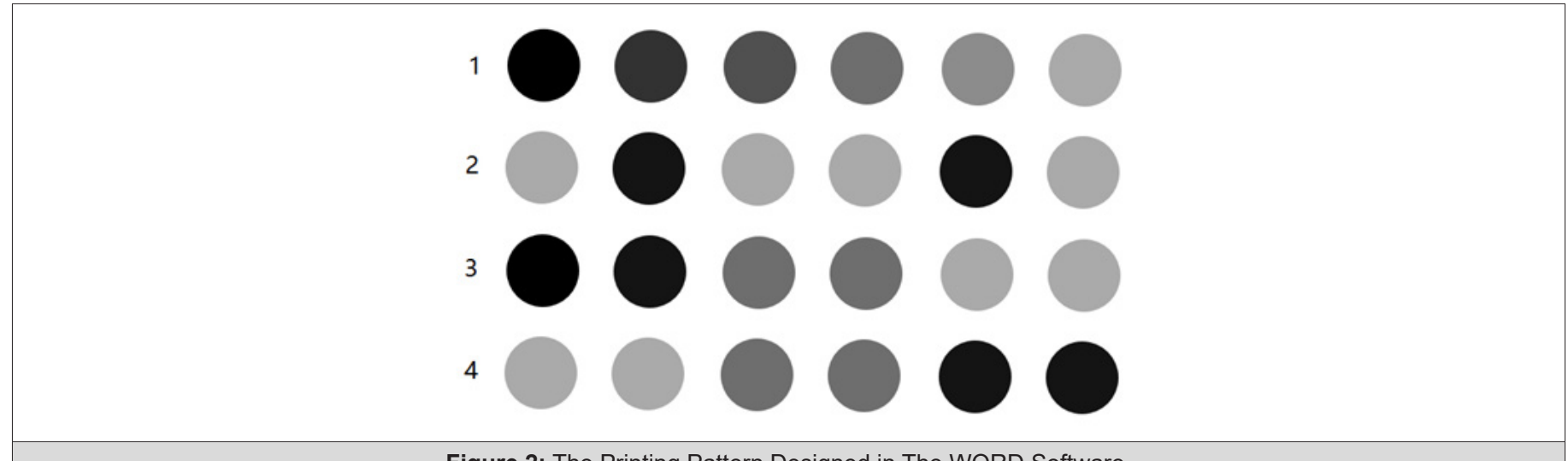

Figure 2: The Printing Pattern Designed in The WORD Software.
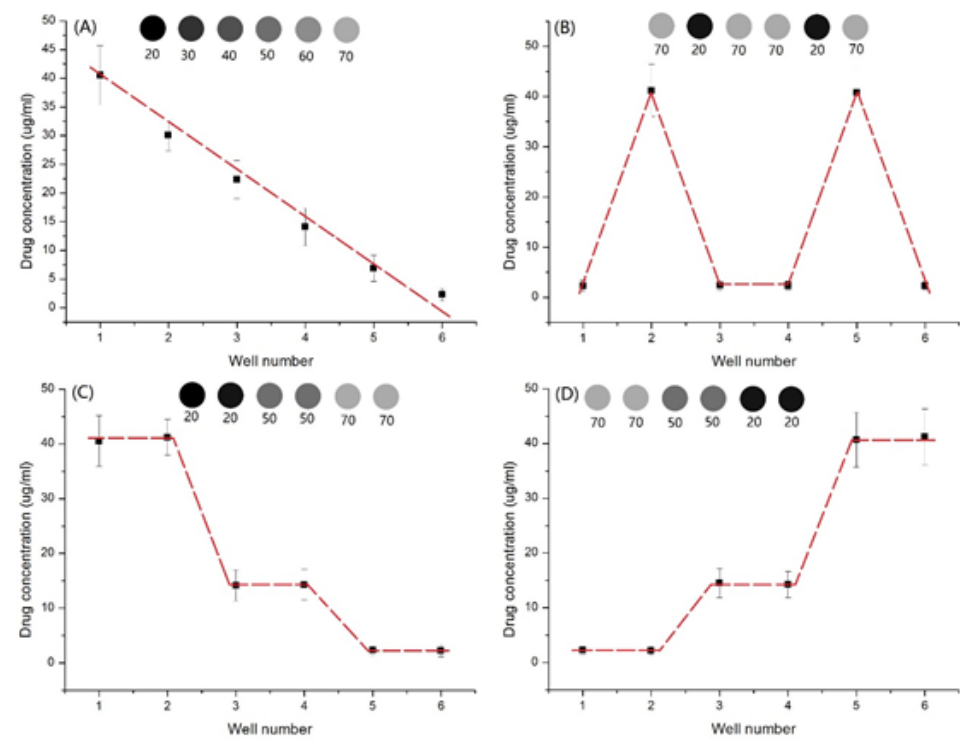

Figure 3: Different Types of Drug Concentration Gradient Printed in the 24-Well Plate.

The printing pattern designed in the WORD software. Each row was designed for generation a specific drug concentration gradient. In the first row, the RGB values from left to right were $(20,20,20)$, $(30,30,30),(40,40,40),(50,50,50),(60,60,60),(70,70,70)$. In the second row, the RGB values from left to right were $(70,70,70)$,
$(20,20,20), \quad(70,70,70),(70,70,70),(20,20,20),(70,70,70)$. In the third row, the RGB values from left to right were $(20,20,20)$, $(20,20,20),(50,50,50),(50,50,50),(70,70,70),(70,70,70)$. In the fourth row, the RGB values from left to right were $(70,70,70)$, $(70,70,70),(50,50,50),(50,50,50),(20,20,20),(20,20,20)$. (Figure 
3) On the top of each plot was the printing pattern designed in the WORD software, and the number below was RGB value. (Figure 4) (from left to right: $3 \times 106 / \mathrm{ml}, 6 \times 106 / \mathrm{ml}, 9 \times 106 / \mathrm{ml}$ ), scale bar: 500 $\mu \mathrm{m}$.

\section{Printing of Mcf-7 Cell Suspension}
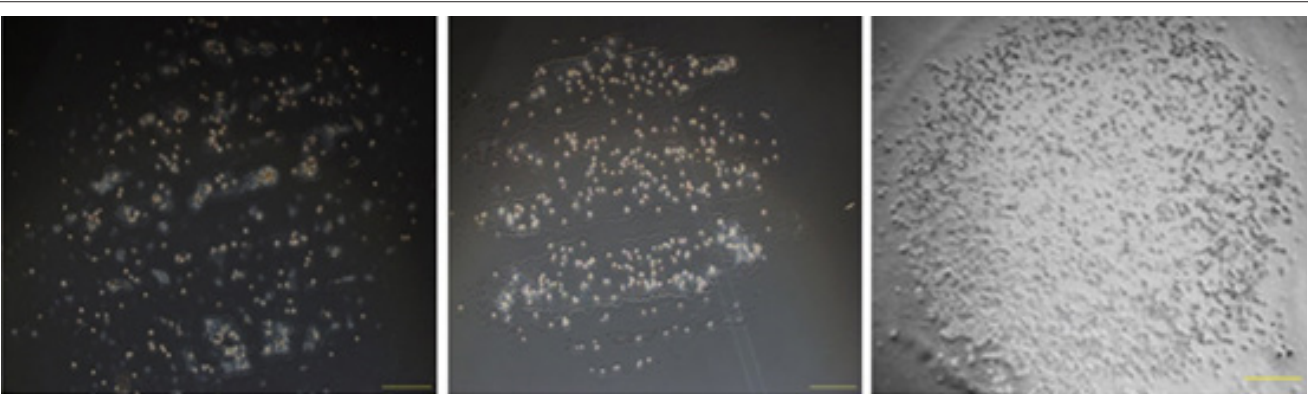

Figure 4: The Bright Field Images of Printed Round Spots of Mcf-7 Cells with Varied Cell Concentration

Figure 4 shows the printed pattern of Mcf-7 cells, with different concentration of Mcf-7 suspension loaded in the printer. When the concentrations were $3 \times 106 / \mathrm{ml}$ and $6 \times 106 / \mathrm{ml}$, the printed round spots were incompletely constructed with sparse distributions of cells. It might be caused by the low concentration of cell suspension. When the concentration was $1.2 \times 107 / \mathrm{ml}$, few inks were injected from the nozzle and the printed round spot was hard to be identified. It might be attributed to the high concentration of the cell suspension which jammed the nozzle. However, when the concentration was $9 \times 106 / \mathrm{ml}$, the printed round spot was full of well-distributed cells. It showed that the cell suspension with a concentration of $9 \times 106 / \mathrm{ml}$ is the best-performed one and should be adopted in the experiment.
After counting, the average number of cells in each round spot in the cell array was $3 \times 104$. After printing, the viability of the cells was the main gauge which reflects the validity of the cell printing process. Hot ink-jet printing was suspected to cause heat damage or mechanical damage on cells [8]. Hoechst33342 is a membrane permissive dye and emits blue fluorescence under $350 \mathrm{~nm}$ excitation after combining with DNA in cells. And PI is only capable of penetrating into dead cells. After PI combines with DNA in cells and emits red fluorescence under $543 \mathrm{~nm}$ excitation. Therefore, we can investigate the viability of cells when coupling Hoechst33342 and PI dyes after cell printing. As seen in Figure 5, the number of dead cells which emit red fluorescence is negligible and it supports that the negative effect of our cell printing technique on the viability of printed cells can be ignored (Figure 5).
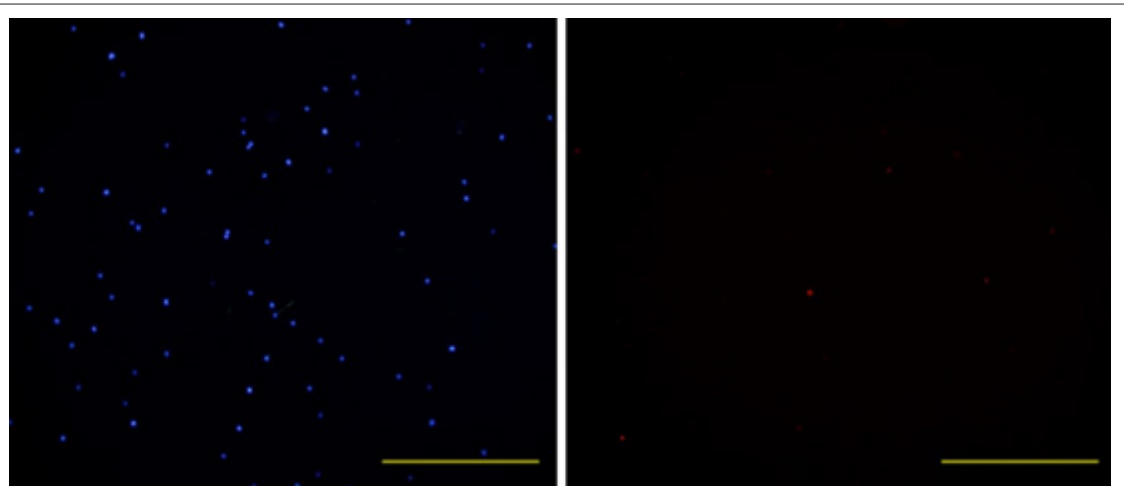

Figure 5: Microscopic observations of printed cells dyed by Hoechst33342(left) and PI (right), under excitations of $350 \mathrm{~nm}$ and $543 \mathrm{~nm}$ wavelengths, respectively. Scale bar: $500 \mu \mathrm{m}$.

\section{Drug Screening Assay Based on Printing}

\begin{tabular}{|c|c|c|c|}
\hline \multicolumn{2}{|c|}{ Table 1: Absorbance by MTT and relative inhibition rate $(\mathrm{n}=3)$. } \\
\hline RGB Value & 5-Fluorouracil Concentration $(\boldsymbol{\mu g} / \mathbf{m l})$ & Average Absorbance \pm Standard Deviation & Relative Inhibition Rate $\mathbf{\%}$ ) \\
\hline 20 & $40.53 \pm 5.18$ & $0.483 \pm 0.02$ & 52.65 \\
\hline 30 & $30.09 \pm 2.69$ & $0.52 \pm 0.03$ & $0.66 \pm 0.03$ \\
\hline 40 & $22.34 \pm 3.31$ & $0.723 \pm 0.02$ & 35.33 \\
\hline 50 & $14.1 \pm 3.29$ & $0.788 \pm 0.04$ & 29.12 \\
\hline 60 & $6.86 \pm 2.26$ & $0.839 \pm 0.05$ & 22.75 \\
\hline 70 & $2.28 \pm 1.08$ & $1.02 \pm 0.03$ & 17.78 \\
\hline
\end{tabular}

The concentration of the printed 5-fluorouracil can be pretuned with the RGB code setup from the WORD software. A Mcf-7 cell/5-fluorouracil composite array was formed after incubation for $48 \mathrm{hrs}$. And the inhibition rate of the experimental group at 
varied concentrations and the control group were tested with MTT method. And the statistics of their optical absorbance at different RGB values and the relative inhibition rates were listed in Table 1. It can be seen from Table 1 that within the range we investigated, the biological viability of the cells in the composite array increased as the RGB value ramped up. In our experimental setup in which RGB code value was kept between 20 and 70, the concentration of the printed 5-fluorouracil increased as the RGB value dropped. In another word, a low RGB value yielded a cell/drug composite array with a relatively high concentration and dose of 5-fluorouracil. Therefore, as the RGB value was lowered, a stronger interaction between Mcf-7 and 5-fluorouracil was achieved, and a higher inhibition phenomenon occurred. This suggested that the Mcf-7 cell/5-fluorouracil composite array established by our method can be used for the inhibition study of 5-fluorouracil at different concentrations and it possessed the potential of evaluating in vitro cellular viability.

\section{Discussion}

Cell printing technique has demonstrated remarkable advantages as following :

a) High precision, cells can be precisely localized and distributed in three dimensions [9];

b) Rapid speed, tissue samples with biological viability can be constructed in a short time;

c) High automation grade, it has the possibility to be industrialized at a large scale [10];

d) Simple to be established and operated, it is straightforward to be modified or upgraded [11].

In our experiment, RGB tuning was used for controlling the output doses of 5 -fluorouracil and the hot inkjet technique was adopted to build cell/drug composite arrays. And the viability of the cells in the composite arrays were evaluated. Inspired by the model from Boland 's group [11,12], a Canon hot inkjet printer and an ink box were successfully modified into a biological printer. And a Mcf7 cell suspension with a concentration of $9 \times 106$ cells $/ \mathrm{ml}$ showed the best printing performance in cell distribution and density on the array. The printed cell array demonstrated patterns with the most complete morphology. By testing the concentration of drug solutions with HPLC, we were able to identify the concentration of 5 -fluorouracil on the printed matrix produced from different RGB code value. The results show that high-definition Mcf-7 cell arrays can be constructed with our modified printer and ink box. And moreover, the concentration of 5-fluorouracil arrays can be tuned by changing the RGB color code value. The viability of cells by printing is the major indicator for the feasibility of the printing operation. We used Hoechst33342 and PI to dye the printed cells and applied excitations of $350 \mathrm{~nm}$ and $543 \mathrm{~nm}$, respectively. The results showed that hot inkjet printing has little damage on the Mcf-7 cells, and they are in consistency with the literatures $[13,14]$. Also, we used MTT method to identify the inhibition rate of 5-fluorouracil on Mcf7 in the cell/drug array. When RGB code value was 30, the dose of the 5 -fluorouracil was $30.09 \pm 2.69 \mu \mathrm{g} / \mathrm{ml}$ with a relative inhibition rate of $49.02 \%$. These results supported the feasibility of applying the cell/drug printing method in investigating in vitro cellular viability and it provides new possibility of drug screening based on biological printing.

\section{Conclusion}

In this study, we developed an automatic method to generate drug concentration gradient in 24-well plate based on hot inkjet printer. The total time was less than 5s. Arbitrary concentration gradient can be easily generated by RGB coding. The cost of printing equipment was very low. This method is expected to make drug screening experiment more user-friendly.

\section{Acknowledgement}

This project was supported by the postdoctoral foundation of China (21675017), the discipline layout project of Shenzhen (JCYJ2018050815247476) and Sanming Project of Medicine in Shenzhen (No. SZSM201612049).

\section{References}

1. Dertinger SKW, Chiu DT, Jeon NL, Whitesides GM (2001) Generation of gradients having complex shapes using microfluidic networks. Anal Chem 73(6): 1240-1246.

2. Ye N, Qin J, Shi W and Lin B (2007) Characterizing doxorubicin-induced apoptosis in HepG2 cells using an integrated microfluidic device. Electrophoresis 28(7): 1146-1153.

3. Ye N, Qin J, Shi W, Liu X, Lin B (2007) Cell-based high content screening using an integrated microfluidic device. Lab on a Chip 7(12): 1696-1704.

4. Guillotin B and Guillemot F (2011) Cell patterning technologies for organotypic tissue fabrication. Trends Biotechnol 29(4): 183-190.

5. Zhigang G, Tingting Z, Jiu D, Xiaorui L, Yueyang Q et al. (2017) Ultrasensitive Detection of Hair Cortisol Based on Portable Raman Spectrometer and Double-layer Paper Microdevice. Acta Chimica Sinica 75(4): 355359.

6. Zheng Q Lu J, Chen H, Huang L, Cai J, et al. (2011) Application of inkjet printing technique for biological material delivery and antimicrobial assays. Anal Biochem 410(2): 171-176.

7. Owczarczak AB, Shuford SO, Wood ST, Deitch S, Dean D (2012) Creating Transient Cell Membrane Pores Using a Standard Inkjet Printer. Jove (61): e3681.

8. Mironov V, Boland T, Trusk T, Forgacs G, Markwald RR (2003) Organ printing: computer-aided jet-based 3D tissue engineering. Trends Biotechnol 21(4): 157-161.

9. Xu T, Zhao WX, Zhu JM, Albanna MZ, Yoo JJ, et al. (2013) Complex heterogeneous tissue constructs containing multiple cell types prepared by inkjet printing technology. Biomaterials 34(1): 130-139.

10. Wilson WC and Boland T (2003) Cell and organ printing 1: Protein and cell printers. Anat Rec Part A 272(2): 491-496.

11. Roth EA, Xu T, Das M, Gregory C, Hickman JJ, et al. (2004) Inkjet printing for high-throughput cell patterning. Biomaterials 25(17): 3707-3715.

12. Cui XF, Dean D, Ruggeri ZM, Boland T (2010) Cell Damage Evaluation of Thermal Inkjet Printed Chinese Hamster Ovary Cells. Biotechnol Bioeng 106(6): 963-969.

13. Xu T, Jin J, Gregory C, Hickman JJ, Boland T (2005) Inkjet printing of viable mammalian cells. Biomaterials 26(1): 93-99.

14. Derby B (2008) Bioprinting: inkjet printing proteins and hybrid cell-containing materials and structures. J Mater Chem 18(47): 5717-5721. 